\title{
Optimal Contract with Moral Hazard for Public Private Partnerships*
}

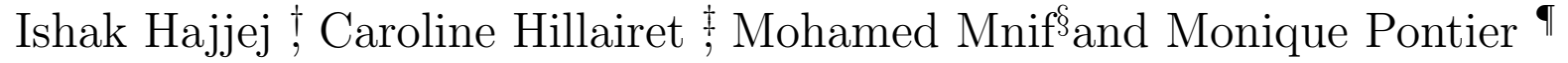

\section{Janvier 2017}

\begin{abstract}
Public-Private Partnership (PPP) is a contract between a public entity and a consortium, in which the public outsources the construction and the maintenance of an equipment (hospital, university, prison...). One drawback of this contract is that the public may not be able to observe the effort of the consortium but only its impact on the social welfare of the project. We aim to characterize the optimal contract for a PPP in this setting of asymmetric information between the two parties. This leads to a stochastic control under partial information and it is also related to principal-agent problems with moral hazard. Considering a wider set of information for the public and using martingale arguments in the spirit of Sannikov [18, the optimization problem can be reduced to a standard stochastic control problem, that is solved numerically. We then prove that for the optimal contract, the effort of the consortium is explicitly characterized. In particular, it is shown that the optimal rent is not a linear function of the effort, contrary to some models of the economic literature on PPP contracts.
\end{abstract}

Keywords : Public Private Partnership, stochastic control under partial information, HJB equation, Moral Hazard.

${ }^{*}$ We thank Guillaume Carlier, Ivar Ekeland, Dylan Possamaï, Nizar Touzi and Stéphane Villeneuve for interesting and helpful discussions and comments.

†University Tunis Manar, ENIT, Lamsin

${ }_{\ddagger}$ ENSAE ParisTech, Crest. The author acknowledges funding from the research programs Chaire Risques Financiers of Fondation du Risque and Investissements d'Avenir (ANR-11-IDEX-0003/Labex Ecodec/ANR-11-LABX-0047).

$\S$ University Tunis Manar, ENIT, Lamsin

『Institut de Mathématiques de Toulouse 


\section{Introduction}

A Public Private Parternship contract is defined by the split between private and public tasks concerning a public services, namely: the design of the project, the construction (building), the financing and the maintenance (operate). DBFO means that all the four tasks are supported by the private partner. The goal of PPP contracts is to transfer the risk to the consortium, to provide a better value for money in the use of public funds. In France, by the law of 2008 endorsing the order of 17th June 2004, PPP contract can not be used except if it is expressly justified with regarded to at least one of the following criteria: emergency, complexity, economic efficiency...and actually the conclusion is that almost all projects are in emergency...

The relevance of outsourcing an investment in order to reduce the debt of a public entity has been studied in Espinosa et al. [8]. Here we do not focus on the cost of the construction but on the maintenance aspect of the PPP contract. Hillairet and Pontier propose in [10] a study on PPP and their relevance, assuming the eventuality of a default of the counterparty. In their model, as in other economic papers such as Iossa et al. [11], the rent is assumed to be a linear rule of the effort of the consortium: although this modelisation leads to tractable computations, it seems very "ad hoc" and economically questionable. The present work does not assume any a priori form for the rent, and in our numerical example, it is shown that the optimal rent is actually not a linear rule.

This paper focuses on the informational asymmetry issue in PPP contracts. Indeed, public and private partners obviously do not share the same information for negotiation, management and follow-up of the contract. Auriol-Picard [1] prove that Build-Operate-Transfer (BOT) contracts (a variant of PPP contracts) may be relevant for the public in case of better information of the private partner, provided a large enough number of concession candidates. But for example in France only three consortium are able to support a PPP contract (Bouygues, Vinci, Eiffage). The support mission of PPP (MAPPP in French, for Mission d'Appui aux PPP), responsible for evaluating the projects in view of legitimate the use of a PPP contract, aims also to reduce the information asymmetry between public entity and consortium. However, as pointed out by the General Inspectorate of Finance in December 2012, the multiple roles of the mission put it "de facto" in a potential situation of conflict of interest.

Besides, the public may not be able to observe the effort of the consortium, but only its impact on the social welfare of the project. Thus characterizing an optimal PPP contract in this setting of asymmetric information between both partners is related to principal-agent problems with moral hazard. As shown in book of Cvitanic et al. [4], a general theory can be used to solve these problems, by means of forward-backward stochastic differential equations. This work is inspired by the literature on dynamic contracting using recursive methods, and in particular the seminal paper of Sannikov [18] (2008). In Biais et al. [3], the agent is risk-neutral and his efforts, unobservable by the principal, reduce the likelihood of large (but relatively infrequent) losses of the size of a project: more precisely, the losses occur according to a Poisson process whose intensity is controlled by the agent. Pagès and Possamaï [15] propose an optimal contracting between competitive investors and an impatient bank monitoring a pool of long-term loans subject to Markov contagion. The unobservable bank monitoring decision 
affects the default intensity of an entity of the pool. Optimal contracting in a Brownian setting with risk-averse agent and principal has also been studied recently in Cvitanic et al. [5], by identifying a family of admissible contracts for which the optimal agent's action is explicitly characterized, and leading to a tractable case for CARA (exponential) utility functions.

In this paper, due to the long maturity of PPP, we consider a perpetual contract between a public entity and a consortium. The consortium supports the initial cost of the project as well as the maintenance costs. The effort that the consortium does to improve the social value of the project is not observable by the public. Thus the rent the public pays to the consortium, to compensate him for his efforts and for the operational costs, is determined on the basis of the public information, that is according to the social value of the project. This is related to principal/agent problem with moral hazard and our approach relies on stochastic control under partial information, as in Bensoussan [2]. We consider a Stackelberg leadership model: the public (the principal) is the leader by offering a contract (characterized by the rent), while the consortium (the agent) gives a best response (characterized by the effort). The aim of this paper is to characterize such optimal contracts. To overcome the difficulty that the control process of the consortium (the effort) is not observable by the public, we restrict the family of admissible contracts to a set of contracts that lead to a tractable characterization of the consortium effort. This could be economically interpreted by the fact that others contracts, for which the public does not know what incentives they will provide to the consortium effort, will likely not be offered. Moreover, we theoretically prove that the optimal contract is indeed of this form. Finally we characterize optimal contracts and provide numerical solutions.

This paper is organized as follows. Section 2 presents the problem, Section 3 provides the solution of this optimal control via Hamilton-Jacobi-Belman equation. Section 4 concludes with numerical illustrations based on the Howard algorithm.

\section{Public Private Partnership's optimal contracts}

Throughout the paper, $\left(\Omega, \mathbb{F}=\left(\mathcal{F}_{t}\right)_{t \in[0, T]}, \mathbb{P}\right)$ is a filtered probability space, with $\mathbb{F}$ a Brownian filtration generated by a standard Brownian motion $W$.

\section{$2.1 \quad$ Effort and rent}

The operational cost $\left(C_{s}\right)_{s \geq 0}$ of the project, supported by the consortium (and not observed by the public), is a non-negative $\mathbb{F}$-adapted process

$$
C_{t}=C_{0}+k t+\sigma W_{t}
$$

where

- $C_{0}>0$ is the initial cost of the project, taking into account the construction of the infrastructure. 
- $C_{t}$ is the cumulative cost of the project over the period $[0, t]$, taking into account both the cost of the construction and the cost of the infrastructure maintenance.

- $k>0$ and $\sigma>0$ are respectively the drift and the volatility of the operational cost of the infrastructure maintenance.

Remark 2.1 The cost process $\left(C_{s}\right)$ is not necessarily non-negative for all s. Nevertheless, as it is proved in Appendix 5, a sufficient condition to get the cost non-negative on time interval $[0, T]$ with at least probability 0.95 is $C_{0} /(\sigma \sqrt{T}) \geq 1.96$.

The consortium supports the operational cost and chooses the effort he does to improve his service for the project : the effort is a non-negative $\mathbb{F}$-adapted process $\left(A_{s}\right)_{s \geq 0}$, it improves the social value of the project. The social welfare, defined as the social value of the project plus the operational cost, is a $\mathbb{F}$-adapted process $\left(X_{s}\right)_{s \geq 0}$ given by

$$
X_{t}:=X_{0}+\int_{0}^{t}\left(\varphi\left(A_{s}\right) d s+d C_{s}\right)=X_{0}+\int_{0}^{t}\left(\varphi\left(A_{s}\right)+k\right) d s+\sigma d W_{s}
$$

where $X_{0}$ is the initial value of the project (i.e. of the construction, it may be a function of $C_{0}$ ) and $\varphi$ is specified hereafter.

The public observes the social value $X$ of the project, but he does not observe directly the effort of the consortium. Thus his information is conveyed by the filtration $\mathbb{F}^{X}$ generated by the social value process $X$. The public chooses the rent he will pay to the consortium to compensate him for his efforts and the operational costs that he supports; the rent is a non-negative $\mathbb{F}^{X}$-adapted process $\left(R_{s}\right)_{s \geq 0}$.

Thus we are looking for optimal control processes $(R, A)$ with $R$ adapted to the filtration generated by the observation $X$ but itself is depending on the control process $A$. Remark that in our model the effort $A$ only affects the drift and not the volatility of the social welfare $X$ (the case of an impact both on the drift and the volatility will be done in a future work). We develop here a strong approach, in the context of stochastic control under partial observation as in Bensoussan [2] Section 2.3.

\section{$2.2 \quad$ A Stackelberg leadership model}

We define the respective optimization problems for the consortium and the public. Due to the long maturity of PPP contract (up to 30-50 years), we assume that the contract is perpetual. The public and the consortium have the same time preference parameter $\delta>0$. Let us first define the functions involved in the formulation of the optimization problems:

\section{Assumption 2.2}

- $U$ is the utility function of the consortium, strictly concave strictly increasing and satisfying $U(0)=0$ and Inada's conditions $U^{\prime}(\infty)=0, U^{\prime}(0)=\infty$. 
- $\varphi$ models the impact of the consortium's efforts on the social value, $\varphi$ is strictly concave increasing satisfying $\varphi(0)=0, \varphi^{\prime}(\infty)=1($ so $\varphi(x) \geq x), \varphi^{\prime}(0)<\infty$.

- $h$ is the cost of the effort for the consortium; $h$ is convex, $h(0)=0, h^{\prime}(0)>0$, (thus $h$ is increasing) and $h^{\prime}(\infty)=\infty$.

- $h \circ \psi^{-1}$ is convex where $\psi:=\frac{1}{2}\left(\frac{h^{\prime}}{\varphi^{\prime}}\right)^{2}$.

Finally, the public does not want to pay a rent over a given amount $\bar{r}$.

Remark 2.3 The function $\frac{h^{\prime}}{\varphi^{\prime}}$ is increasing positive, and $\psi(e)=\frac{1}{2}\left(\frac{h^{\prime}}{\varphi^{\prime}}(e)\right)^{2} \geq \frac{1}{2}\left(\frac{h^{\prime}(0)}{\varphi^{\prime}(0)}\right)^{2}>0$.

We define different sets of admissible contracts, depending on the information flow:

$$
\begin{aligned}
\mathcal{A}:= & \left\{\left(R_{s}, A_{s}\right)_{s \geq 0} \quad \mathbb{F} \text {-adapted, } A_{s} \geq 0, d s \otimes d \mathbb{P}\right. \text { a.e. such that } \\
& \left.U\left(R_{s}-k\right)-h\left(A_{s}\right) \geq 0 d s \otimes d \mathbb{P} \text { a.e. and } k \leq R_{s} \leq \bar{r},\right\} . \\
\mathcal{A}^{X}:= & \mathcal{A} \cap\left\{\left(R_{s}, A_{s}\right)_{s \geq 0}, \text { such that } \quad R \text { is } \mathbb{F}^{X} \text {-adapted }\right\} .
\end{aligned}
$$

Those admissibility conditions ensure that entering into the contract provides a non-negative value for the consortium. Remark that $(R, A)$ in $\mathcal{A}$ implies the following integrability properties

$$
e^{-\delta s}\left(\varphi\left(A_{s}\right)-R_{s}+k\right)^{-} \in L^{1}\left(\mathbb{R}^{+} \times \Omega\right), e^{-\delta s}\left(U\left(R_{s}-k\right)-h\left(A_{s}\right)\right) \text { and } \frac{h^{\prime}}{\varphi^{\prime}}\left(A_{s}\right) \in L_{l o c}^{2}\left(\mathbb{R}^{+} \times \Omega\right) .
$$

We consider a Stackelberg leadership model: the public is the leader by offering a contract (characterized by the rent process $R$ ). The consortium gives a best response in terms of the effort process $\left(A_{s}\right)_{s \geq 0}$.

Objective function and continuation value for the consortium and for the public: The consortium aims to optimize the expectation of his aggregate utility of the rent minus the drift of the operational cost, minus the cost of his effort

$$
A \rightarrow J_{0}^{C}(R, A)=\mathbb{E}\left(\int_{0}^{\infty} e^{-\delta s}\left(U\left(R_{s}-k\right)-h\left(A_{s}\right)\right) d s\right) .
$$

The public anticipates the consortium's best response to propose the optimal contract and aims to optimize the expectation of the social welfare minus the rent paid to the consortium

$$
\begin{aligned}
R \rightarrow J_{0}^{P}(R, A) & =\mathbb{E}\left(\int_{0}^{\infty} e^{-\delta s}\left(d X_{s}-R_{s} d s\right)\right) \\
& \left.=\mathbb{E}\left(\int_{0}^{\infty} e^{-\delta s}\left(d C_{s}+\varphi\left(A_{s}\right) d s\right)-R_{s} d s\right)\right) \\
& =\mathbb{E}\left(\int_{0}^{\infty} e^{-\delta s}\left(\varphi\left(A_{s}\right)-R_{s}+k\right) d s\right),
\end{aligned}
$$


the last equality being a consequence of the dynamics of the social welfare $X$ and the fact that $\mathbb{E}\left(\int_{0}^{\infty} e^{-\delta t} \sigma d W_{t}\right)=0$. According to $(2.3)$, the integrals of both objective functions equation (2.4) and equation (2.5) are well defined. From a dynamic point of view, the objective function at time $t$ for the consortium is $\mathbb{P}$-a.s.

$$
J_{t}^{C}(R, A):=\mathbb{E}\left(\int_{t}^{\infty} e^{-\delta(s-t)}\left(U\left(R_{s}-k\right)-h\left(A_{s}\right)\right) d s \mid \mathcal{F}_{t}\right)
$$

while the objective function at time $t$ for the public is $\mathbb{P}$-a.s.

$$
J_{t}^{P}(R, A):=\mathbb{E}\left(\int_{t}^{\infty} e^{-\delta(s-t)}\left(\varphi\left(A_{s}\right)-R_{s}+k\right) d s \mid \mathcal{F}_{t}^{X}\right)
$$

The consortium chooses the effort $A$ and the public chooses the rent $R$ in the set of admissible contracts $\mathcal{A}^{X}$, such as to optimize their respective objective functions, leading to the corresponding continuation value process denoted respectively $V^{C}$ and $V^{P}$.

More precisely, an effort process $A$ is incentive compatible with respect to a given rent $R$ if it optimizes the consortium's expected utility (defined in equation (2.4)) given $R$. The problem of the public is to find $R$ (the contract) that optimizes his expected discounted profit (defined in equation (2.5), given the corresponding incentive compatible effort $A$.

Compared to a classic optimization control problem, the difficulty of our formulation is that the public does not observe the control $A$ of the consortium, but he observes only its impact on the social value $X$ which is the state process of the optimization control problem. The state process $X$ appears in an implicit way in the formulation of the optimization problem of the consortium, through the rent process $R$, control of the public. Thus there is no explicit link between the two controls $A$ and $R$, the only indirect link involves the state process $X$. The trick to overcome this difficulty is to reformulate the optimization problems in terms of the consortium continuation value process $V^{C}$.

\subsection{Incentive compatible contract}

To encourage the consortium to follow the recommended effort, the public proposes an incentive compatible contract. This subsection characterizes the incentive compatible contracts for $(R, A)$ in $\mathcal{A}$ the largest set of admissibility. As in Sannikov [18] or Cvitanic et al. [5] in a weak formulation setting, the following Proposition 2.4 characterizes the dynamics of consortium continuation value process. It is coherent with the result of [5], proved using BSDE's technics, in a more general framework.

Proposition 2.4 If the contract $(R, A) \in \mathcal{A}$ is incentive compatible, with $A$ taking value in ] $0, \bar{a}\left[\right.$ with $\bar{a}:=h^{-1} \circ U(\bar{r}-k)$, then the dynamics of the consortium objective function is

$$
d J_{t}^{C}(R, A)=\delta J_{t}^{C}(R, A) d t-\left(U\left(R_{t}-k\right)-h\left(A_{t}\right)\right) d t+Y_{t} d W_{t}, \quad \text { a.s. }
$$


where

$$
Y_{t}=\sigma \frac{h^{\prime}\left(A_{t}\right)}{\varphi^{\prime}\left(A_{t}\right)}>0
$$

Therefore, $A_{t}=\left(\frac{h^{\prime}}{\varphi^{\prime}}\right)^{-1}\left(Y_{t} \sigma^{-1}\right)$, denoted as $A^{*}\left(Y_{t}\right)$, realizes the optimal value in (2.6) for the consortium. If $R$ is the optimal rent for the public, then the corresponding incentive compatible effort takes value in ]0, $\bar{a}$ [ and the incentive compatible dynamic of the consortium continuation value process is

$$
d V_{t}^{C}=\delta V_{t}^{C} d t-\left(U\left(R_{t}-k\right)-h\left(A_{t}\right)\right) d t+\sigma \frac{h^{\prime}\left(A_{t}\right)}{\varphi^{\prime}\left(A_{t}\right)} d W_{t}
$$

Remark 2.5 Incentive compatible contracts imply that the effort $A$ is necessarily defined as an $\mathbb{F}^{V^{C}}=\left(\sigma\left(V_{s}^{C}, s \leq t\right)_{t \geq 0}\right.$ adapted process since it has to satisfy $A_{t}=\left(\frac{h^{\prime}}{\varphi^{\prime}}\right)^{-1}\left(Y_{t} \sigma^{-1}\right)$ where $Y_{t}^{2}=\frac{d}{d t}\left\langle V^{C}\right\rangle_{t}$

Proof: For any admissible pair $(R, A) \in \mathcal{A}$, let us define the process

$$
M_{t}^{C}(R, A):=e^{-\delta t} J_{t}^{C}(R, A)+\int_{0}^{t} e^{-\delta s}\left(U\left(R_{s}-k\right)-h\left(A_{s}\right)\right) d s, t \geq 0 .
$$

The pair $(R, A) \in \mathcal{A}, U\left(R_{s}-k\right)-h\left(A_{s}\right)$ takes its values in the bounded interval $[0, U(\bar{r}-k)]$. Therefore $M^{C}$ is an $\mathbb{F}$-martingale, uniformly integrable, as an $\mathbb{F}$-conditional expectation of a bounded random variable: there exists a $\mathbb{F}$-predictable process $Y$ such that $e^{-\delta s} Y_{s} \in L^{2}\left(\Omega \times \mathbb{R}^{+}\right)$ and for any $t$ :

$$
e^{-\delta t} J_{t}^{C}(R, A)+\int_{0}^{t} e^{-\delta s}\left(U\left(R_{s}-k\right)-h\left(A_{s}\right)\right) d s=J_{0}^{C}(R, A)+\int_{0}^{t} e^{-\delta s} Y_{s} d W_{s} .
$$

The boundedness of $U\left(R_{s}-k\right)-h\left(A_{s}\right)$ implies that the process $e^{-\delta t} J_{t}^{C}(R, A)$ is uniformly bounded by the integral $\int_{t}^{\infty} e^{-\delta s} d s$ which goes to 0 when $t$ goes to infinity. Thus $t$ going to infinity in 2.10 leads to the consortium's objective value

$$
\int_{0}^{\infty} e^{-\delta s}\left(U\left(R_{s}-k\right)-h\left(A_{s}\right)\right) d s-\int_{0}^{\infty} e^{-\delta s} Y_{s} d W_{s}=J_{0}^{C}(R, A) .
$$

Using Definition (2.2)

$$
J_{0}^{C}(R, A)=\int_{0}^{\infty} e^{-\delta s}\left(U\left(R_{s}-k\right)+Y_{s} \sigma^{-1}\left(\varphi\left(A_{s}\right)+k\right)-h\left(A_{s}\right)\right) d s-\int_{0}^{\infty} e^{-\delta s} \sigma^{-1} Y_{s} d X_{s}
$$

The public observes the social value $\left(X_{t}\right)_{t \geq 0}$ but he could not make difference between the effort $\left(A_{t}\right)_{t \geq 0}$ and the Brownian motion $\left(W_{t}\right)_{t \geq 0}$. The consortium knows that the incentive contract proposed by the public does not optimize the integral $\int_{0}^{\infty} e^{-\delta s} \sigma^{-1} Y_{s} d X_{s}$. In order to motivate the consortium, the public proposes a contract such that the corresponding optimal 
effort $\left(A_{t}^{*}\right)_{t \geq 0}$ maximizes the concave function $a \rightarrow Y_{t} \sigma^{-1} \varphi(a)-h(a)$, for all $t, d t \otimes d \mathbb{P}$ almost everywhere.

It remains to prove that, for the "optimal" contract, the optimum on $[0, \bar{a}]$ of this function is not attained on the bounds of the interval (where $\bar{a}:=h^{-1} \circ U(\bar{r}-k)$ is the upper bound of the effort). More precisely, we prove that if the incentive compatible effort is equal to 0 or to $\bar{a}$, then the public could propose a better contract (that is a rent) that will increase his value function.

Let $t \geq 0$ and $\eta>0$ be fixed and consider the stochastic set $D_{t, \eta}:=\left\{\omega: A_{s}^{*}(\omega)=0\right.$ for $s \in$ $[t, t+\eta]\}$. From the definition of the public continuation value process, $R^{*}=k$ on $D_{t, \eta}$. Therefore, on this set, $V^{C}$ is a constant process. Since the dynamics of the consortium continuation value process follows $d V_{s}^{C}=\delta V_{s}^{C} d s+Y_{s} d W_{s}$, on $D_{t, \eta}$, the uniqueness of the Itô decomposition implies $V^{C}=Y=0$ on $D_{t, \eta} d t \otimes d \mathbb{P}$ a.e. On the other hand, on $D_{t, \eta}, A=0, R=k$ implies $V_{s}^{P}=0$. However, since the public is the leader, he could propose a rent to the consortium satisfying $R_{s}-k=U^{-1}\left(h\left(A_{s}\right)\right)$. The concavity of the functions $\varphi, h^{-1}, U$, yields the function $g: x \rightarrow \varphi\left[h^{-1}(U(x))\right]-x$ is concave. Moreover it satisfies $g(0)=0, g^{\prime}(0)=+\infty$ and going to $-\infty$ when $x \rightarrow \infty$, thus $\sup _{x \geq 0}\left(\varphi\left[h^{-1}(U(x)]\right)-x\right)>0$ and $V_{s}^{P}>0$. This is a contradiction and $\mathbb{P}\left(D_{t, \eta}\right)=0$. This shows that the incentive compatible effort for the "optimal" contract satisfies $A_{t}^{*}>0 d t \otimes d \mathbb{P}$. Similarly $A_{t}^{*}<\bar{a} d t \otimes d \mathbb{P}$.

In the following, all admissible contracts $(R, A)$ are assumed incentive compatible, and thus are denoted $\left(R, A^{*}(Y)\right)$. With a slight abuse of notation we keep the same notations $\mathcal{A}$ and $\mathcal{A}^{X}$. In the next section, we will first solve the problem under the set of controls $\mathcal{A}$, that is with no restriction of measurability on the rent process. As suggested by Remark 2.5, we will then check that the optimal controls $\left(R^{*}, A^{*}\right)$ over $\mathcal{A}$ are functions of the consortium continuation value $V^{C}$. Thus equation 2.9) modeling the dynamics of the consortium value function is a Markovian diffusion, the solution of which being defined up to its explosion time $\tau$. We will prove that $\tau=+\infty$ a.s.

The public value at time 0 over the class $\mathcal{A}$ is written as follows

$$
v(x):=\sup _{\left(R, A=A^{*}(Y)\right) \in \mathcal{A}} J_{0}^{P}(R, A)=\sup _{\left(R, A=A^{*}(Y)\right) \in \mathcal{A}} E_{x}\left[\int_{0}^{\infty} e^{-\delta s}\left(\varphi\left(A_{s}\right)-R_{s}+k\right) d s\right]
$$

where $E_{x}$ is the conditional expectation with respect to the event $\left\{V_{0}^{C}=x\right\}$. The fact that the effort $A=A^{*}(Y)$ is the best response of the consortium, for a given rent $R$, follows from the incentive compatible dynamic 2.9 of the state process $V^{C}$.

The next section characterizes, through a HJB equation, the function $v$ that realizes the optimum for the public over the class of $\mathcal{A}$. But, actually we will prove that the optimal processes over the class $\mathcal{A}$ are in fact $\mathbb{F}^{X}$-adapted and the optimal value function for the public over the class $\mathcal{A}^{X}$ is indeed $v\left(V_{0}^{C}\right)$ (see Proposition 3.7). Thus we solve ultimately the original optimization problem over the class $\mathcal{A}^{X}$. 


\section{Optimal controls and value functions for the public and the consortium}

We first solve the optimization problem 2.12 over the set of controls $\mathcal{A}$, that is done using the dynamic programming principle. Subsection 3.1 gives a formal derivation of the Hamilton Jacobi Bellman equation

$$
\sup _{(r, a) \in \mathcal{C}}\left[-\delta w(x)+\mathcal{L}^{r, a} w(x)-r+\varphi(a)+k\right]=0,
$$

where the second order differential operator $\mathcal{L}^{r, a}$ is defined by

$$
\mathcal{L}^{r, a} w(x):=w^{\prime}(x)(\delta x-U(r-k)+h(a))+\frac{1}{2} w^{\prime \prime}(x)\left(\sigma \frac{h^{\prime}}{\varphi^{\prime}}(a)\right)^{2}
$$

and the control space $\mathcal{C}$ is defined by

$$
\mathcal{C}:=\left\{(r, a) \in[k, \bar{r}] \times \mathbb{R}^{+}, h(a) \leq U(r-k)\right\} .
$$

Subsection 3.2 relates the HJB equation to the optimization problem (2.12), and characterizes the optimal controls and value functions over $\mathcal{A}$. We then prove in Subsection 3.3 that the optimal controls over the larger set $\mathcal{A}$ are actually in $\mathcal{A}^{X}$.

\subsection{Formal derivation of the HJB equation}

We assume that the public value function $v$ (defined in equation (2.12) ) is of class $C^{2}$. Standard methods (e.g. El Karoui [7] or Pham [16]) are used to provide the HJB equation that is satisfied by the public value function $v$. We give nevertheless some details for the sake of completeness. Let $\eta>0$, and $\left(R, A^{*}(Y)\right) \in \mathcal{A}$, the dynamic programming principle yields

$$
v(x) \geq \mathbb{E}_{x}\left[\int_{0}^{\eta} e^{-\delta s}\left(\varphi\left(A_{s}^{*}(Y)\right)-R_{s}+k\right) d s+e^{-\delta \eta} v\left(V_{\eta}^{C}\right)\right] .
$$

Itô's formula applied to the process $\left(e^{-\delta s} v\left(J_{s}^{C}\left(R, A^{*}(Y)\right)\right)\right)_{s \geq 0}$ and taking expectation yield

$$
0 \geq \mathbb{E}_{x}\left[\int_{0}^{\eta} e^{-\delta s}\left(-\delta v(x)+\mathcal{L}^{R_{s}, A_{s}^{*}(Y)} v\left(J_{s}^{C}\right)+\varphi\left(A_{s}^{*}(Y)\right)-R_{s}+k\right) d s\right] .
$$

Dividing by $\eta$, letting $\eta$ goes to 0 , then using the continuity of the integrands and the mean value theorem we obtain

$$
-\delta v(x)+v^{\prime}(x)(\delta x-U(r-k)+h(a))+\frac{1}{2} v^{\prime \prime}(x)\left(\sigma \frac{h^{\prime}(a)}{\varphi^{\prime}(a)}\right)^{2}-r+\varphi(a)+k \leq 0 .
$$

Since this holds for any control $(r, a) \in \mathcal{C}$, we obtain the inequality

$$
\sup _{(r, a) \in \mathcal{C}}\left[-\delta v(x)+\mathcal{L}^{r, a} v(x)-r+\varphi(a)+k\right] \leq 0 .
$$


On the other hand, suppose that $\left(R^{*}, A^{*}(Y)\right)$ is an optimal control for the class $\mathcal{A}$. Then the dynamic programming principle yields

$$
v(x)=\mathbb{E}_{x}\left[\int_{0}^{\eta} e^{-\delta s}\left(\mathcal{L}^{R_{s}^{*}, A_{s}^{*}(Y)} v\left(J_{s}^{C}\right)+\varphi\left(A_{s}^{*}(Y)\right)-R_{s}^{*}+k\right) d s\right]
$$

where $V_{s}^{C}$ is the consortium value function at time $s$, given by the optimal control $\left(R^{*}, A^{*}(Y)\right)$. By similar arguments, dividing by $\eta$, and sending $\eta$ to 0 , one has at time $t=0$

$$
-\delta v(x)+\mathcal{L}^{R_{0}^{*}, A_{0}^{*}(Y)} v(x)+\varphi\left(A_{0}^{*}(Y)\right)-R_{0}^{*}+k=0
$$

which combined with 3.3 yields 3.1 .

A useful result to solve this HJB equation is the following.

Lemma 3.1 The function $v$ defined in 2.12 is a non-negative bounded function on $\mathbb{R}^{+}$.

Proof: For all $(R, A) \in \mathcal{A}$ and for any $t \geq 0, J_{t}^{C}(R, A) \geq 0$ thus $V_{t}^{C} \geq 0$. Therefore the function $v$ is defined on $\mathbb{R}^{+}$.

For any $(r, a) \in \mathcal{C}, h(a) \leq U(r-k)$ and due to the concavity of $\varphi, \varphi(a)-(r-k) \leq \varphi^{\prime}(0) h^{-1}(U(r-$ $k))-(r-k)$. Since the function $x \rightarrow \varphi^{\prime}(0) h^{-1}(U(x))-x$ is concave, going from 0 at $x=0$ to $-\infty$ when $x \rightarrow \infty$, it admits the maximum

$$
\sup _{x \geq 0}\left(\varphi^{\prime}(0) h^{-1}(U(x))-x\right)
$$

which bounds $v$ from above on $\mathbb{R}^{+}$. Besides, the constant control $R_{t}=k ; A_{t}=0, d t \otimes d \mathbb{P}$, a.e., is admissible and incentive compatible (the best consortium's response to a minimum rent is zero effort). Using this control implies that $\forall x \geqslant 0, v(x) \geq 0$.

\subsection{Verification theorem}

In this subsection the optimal control problem $(2.12)$ is characterized as the solution of the HJB equation (3.1) via a verification theorem (cf. for instance El Karoui [7], Pham [16], Krylov [12] or Fleming-Rishel [9]). The following proposition provides the structure of the optimal control.

Proposition 3.2 Suppose Assumption 2.2, then there exists a unique admissible optimal pair $\left(r^{*}, a^{*}\right)$ which realizes the maximum in HJB equation (3.1). This optimal pair is defined by $r^{*}(x)=\left(k+\left(U^{\prime}\right)^{-1}\left(\frac{-1}{w^{\prime}(x)}\right)\right) \mathbf{1}_{w^{\prime}(x)<0} \wedge \bar{r}$ and $a^{*}(x)=\arg \max \left(a \rightarrow w^{\prime}(x) h(a)+w^{\prime \prime}(x) \sigma^{2} \psi(a)+\varphi(a)\right)$ in the compact interval $\left[0, h^{-1} \circ U\left(\left(U^{\prime}\right)^{-1}\left(\frac{-1}{w^{\prime}(x)}\right) \mathbf{1}_{w^{\prime}(x)<0} \wedge(\bar{r}-k)\right)\right]$. Moreover, the function $r^{*}$ is continuous on $\mathbb{R}^{+}$and $a^{*}$ is Borel measurable on $\left[0, h^{-1} \circ U\left(\left(U^{\prime}\right)^{-1}\left(\frac{-1}{w^{\prime}(x)}\right) \mathbf{1}_{w^{\prime}(x)<0} \wedge(\bar{r}-k)\right)\right]$. 
Example 3.3 For instance, the hypotheses of Proposition 3.2 are satisfied for: $\varphi(x)=x+$ $\ln (1+x), h(x)=\frac{2}{3} \sqrt{1+x}(x+4)-\frac{8}{3}$, so $h^{\prime}(x)=\frac{2+x}{\sqrt{1+x}}, \varphi^{\prime}(x)=1+\frac{1}{1+x}, 2 \psi(x)=1+x$, $\psi^{-1}(x)=2 x-1$. The functions $\varphi$ and $\psi$ are concave, and $\psi^{-1}$ is convex. Finally $h "(x)=$ $\frac{1}{\sqrt{1+x}}-\frac{2+x}{2(1+x)^{3 / 2}}=\frac{x}{2(1+x)^{3 / 2}} \geq 0, h$ is convex, $h(0)=0, h^{\prime}(0)=2, h$ convex yields $h(x) \geq 2 x$.

In this example, the coefficient of $w^{\prime \prime}(x)$ is $\frac{1}{2} \sigma^{2}(1+a)$, so it does not depend on $x$.

Proof: (i) Change of variables:

$$
P=h(a)-U(r-k) ; Q=\frac{1}{2}\left(\frac{h^{\prime}(a)}{\varphi^{\prime}(a)}\right)^{2}=\psi(a) .
$$

Recall $\psi: x \rightarrow \frac{1}{2}\left(\frac{h^{\prime}(x)}{\varphi^{\prime}(x)}\right)^{2}$ is non decreasing since $h$ is convex and $\varphi$ concave. This change of variable is a bijection

$$
[k, \bar{r}] \times \mathbb{R}_{+} \rightarrow[-U(\bar{r}-k), 0] \times \mathbb{R}_{+} ;(r, a) \mapsto(h(a)-U(r-k), \psi(a)) .
$$

with inverse

$$
[-U(\bar{r}-k), 0] \times[\psi(0), \psi(\infty)) \rightarrow[k, \bar{r}] \times \mathbb{R}_{+} ;(P, Q) \mapsto\left(k+U^{-1}\left[h \circ \psi^{-1}(Q)-P\right], \psi^{-1}(Q)\right) .
$$

The constraint $P \leq 0 \Leftrightarrow U(r-k)-h(a) \geq 0$ is the admissibility condition on control $(r, a)$. Thus the HJB equation (3.1) is equivalent to

$\left.\sup _{-U(\bar{r}-k) \leq P \leq 0, \psi(0) \leq Q \leq \psi(\infty)}\left[w^{\prime}(x) P+w^{\prime \prime}(x) \sigma^{2} Q+\varphi\left(\psi^{-1}(Q)\right)-U^{-1}\left(h \circ \psi^{-1}(Q)-P\right)\right)\right]=-\delta x w^{\prime}(x)+\delta w(x)$.

(ii) For any $C^{2}$ function $f$ and under the hypotheses of Proposition 3.2, the function

$$
\mathcal{H}:(P, Q) \mapsto f^{\prime}(x) P+f^{\prime \prime}(x) \sigma^{2} Q+\varphi\left(\psi^{-1}(Q)\right)-U^{-1}\left(h \circ \psi^{-1}(Q)-P\right)+k
$$

is strictly concave.

To prove that $\mathcal{H}$ is strictly concave, we check that $D^{2}(-\mathcal{H})(\mathcal{P}, \mathcal{Q})$ is a positive-definite matrix:

$$
\partial_{P, P}^{2} \mathcal{H}<0 \text {, and } \partial_{Q, Q}^{2} \mathcal{H}<0 \text { and } \partial_{P, P}^{2} \mathcal{H} \partial_{Q, Q}^{2} \mathcal{H}-\left(\partial_{P, Q}^{2} \mathcal{H}\right)^{2}>0
$$

Computing the first order derivatives:

$$
\begin{gathered}
\partial_{P} \mathcal{H}=\left(U^{-1}\right)^{\prime}\left(h \circ \psi^{-1}(Q)-P\right)+f^{\prime}(x), \\
\partial_{Q} \mathcal{H}=\varphi^{\prime}\left(\psi^{-1}(Q)\right)\left(\psi^{-1}\right)^{\prime}(Q)-\left(U^{-1}\right)^{\prime}\left(h \circ \psi^{-1}(Q)-P\right) \cdot\left(h \circ \psi^{-1}\right)^{\prime}(Q)+\sigma^{2} f^{\prime \prime}(x) .
\end{gathered}
$$

Using the concavity of function $U$ the second order derivative $\partial_{P, P}^{2} \mathcal{H}$ satisfies

$$
\left.\partial_{P, P}^{2} \mathcal{H}=-\left(U^{-1}\right) "\left(h \circ \psi^{-1}(Q)-P\right)\right)<0 .
$$


Other concavity arguments yield

$$
\begin{aligned}
\partial_{Q, Q}^{2} \mathcal{H} & =\left(\psi^{-1}\right)^{\prime \prime}(Q) \varphi^{\prime} \circ \psi^{-1}(Q)+\left(\left(\psi^{-1}\right)^{\prime}(Q)\right)^{2} \varphi^{\prime \prime} \circ\left(\psi^{-1}\right)(Q) \\
& -\left(h \circ \psi^{-1}\right) "(Q)\left(U^{-1}\right)^{\prime}\left(h \circ \psi^{-1}(Q)-P\right) \\
& -\left(\left(h \circ \psi^{-1}\right)^{\prime}(Q)\right)^{2}\left(U^{-1}\right)^{\prime \prime}\left(h \circ \psi^{-1}(Q)-P\right)<0 .
\end{aligned}
$$

Finally

$$
\partial_{P, Q}^{2} \mathcal{H}=\left(h \circ \psi^{-1}\right)^{\prime}(Q)\left(U^{-1}\right) "\left(h \circ \psi^{-1}(Q)-P\right)
$$

and since $\left(U^{-1}\right) "\left(h \circ \psi^{-1}(Q)-P\right)>0$, the Jacobian sign is the one of

$$
-\left(\psi^{-1}\right) "(Q) \varphi^{\prime} \circ \psi^{-1}(Q)-\left(\left(\psi^{-1}\right)^{\prime}(Q)\right)^{2} \varphi^{\prime \prime} \circ\left(\psi^{-1}\right)(Q)+\left(h \circ \psi^{-1}\right) "(Q)\left(U^{-1}\right)^{\prime}\left(h \circ \psi^{-1}(Q)-P\right)
$$

which is positive (using again concavity arguments). Then

$$
\partial_{P, P}^{2} \mathcal{H} \partial_{Q, Q}^{2} \mathcal{H}-\left(\partial_{P, Q}^{2} \mathcal{H}\right)^{2}>0
$$

and $\mathcal{H}$ is strictly concave.

(iii) Existence of an optimal pair $\left(r^{*}, a^{*}\right)$.

In the HJB equation one has to maximize the function

$$
g: r \mapsto-w^{\prime}(x) U(r-k)-r .
$$

When $w^{\prime}(x) \geq 0$, this function is non-increasing and the optimum is $k$.

Otherwise, the function is concave and the optimum is achieved for

$$
r^{*}(x)=\left(k+\left(U^{\prime}\right)^{-1}\left(\frac{-1}{w^{\prime}(x)}\right) \mathbf{1}_{w^{\prime}(x)<0}\right) \wedge \bar{r} .
$$

Moreover $x \rightarrow r^{*}(x)$ is continuous even at zero points of the function $w^{\prime}$ because $\left(U^{\prime}\right)^{-1}(\infty)=0$. The optimal pair has to satisfy the admissibility condition

$$
0 \leq h\left(a^{*}\right) \leq U\left(\left(U^{\prime}\right)^{-1}\left(\frac{-1}{w^{\prime}(x)}\right) \mathbf{1}_{w^{\prime}(x)<0} \wedge(\bar{r}-k)\right) .
$$

Since the function

$$
a \mapsto w^{\prime}(x) h(a)+w^{\prime \prime}(x) \sigma^{2} \psi(a)+\varphi(a)
$$

is continuous on the compact interval $\left[0, h^{-1} \circ U\left(\left(U^{\prime}\right)^{-1}\left(\frac{-1}{w^{\prime}(x)}\right) \mathbf{1}_{w^{\prime}(x)<0} \wedge(\bar{r}-k)\right)\right]$ there exists an optimal solution $a^{*}$. Moreover by a selection theorem (See Appendix B, Fleming and Rishel $[9]$ ), there exists a Borel-measurable function $a^{*}$ from $\left[0, h^{-1} \circ U\left(\left(U^{\prime}\right)^{-1}\left(\frac{-1}{w^{\prime}(x)}\right) \mathbf{1}_{w^{\prime}(x)<0} \wedge(\bar{r}-k)\right)\right]$ into $\mathbb{R}$. Finally, by construction $\left(r^{*}, a^{*}\right)$ takes its values in $\mathcal{C}$.

Proposition 3.4 Define $\bar{x}:=\frac{1}{\delta}\left(U o\left(U^{\prime}\right)^{-1}\left(\frac{h^{\prime}(0)}{\varphi^{\prime}(0)}\right)\right)$ and suppose Assumption 2.2. Then

(i) The consortium initial value $V_{0}^{C}$ is in the interval $[0, \bar{x}]$.

(ii) $v(\bar{x})=0$. 
Proof: i) We assume $V_{0}^{C}>\frac{1}{\delta}\left(U o\left(U^{\prime}\right)^{-1}\left(\frac{h^{\prime}(0)}{\varphi^{\prime}(0)}\right)\right)$ and we prove that it leads to a contradiction. Let $r_{0}:=k+U^{-1}\left(\delta V_{0}^{C}\right)$ and define the strict convex function $\tilde{h}():.=h()-.U^{\prime}\left(r_{0}-k\right) \varphi($.$) . Then$ $\delta V_{0}^{C}>U o\left(U^{\prime}\right)^{-1}\left(\frac{h^{\prime}(0)}{\varphi^{\prime}(0)}\right), U$ increasing and $U^{\prime}$ decreasing imply that $U^{\prime}\left(r_{0}-k\right) \varphi^{\prime}(0)<h^{\prime}(0)$. For any control $\left(R, A^{*}(Y)\right) \in \mathcal{A}$, one has by strict concavity of the function $U$

$$
U\left(R_{s}-k\right)<U\left(r_{0}-k\right)+U^{\prime}\left(r_{0}-k\right)\left(R_{s}-r_{0}\right), \quad d s \otimes d \mathbb{P} \quad \text { a.e. when } R_{s} \neq r_{0} .
$$

and by strict convexity of $\tilde{h}():.=h()-.U^{\prime}\left(r_{0}-k\right) \varphi($.$) , with our choice of r_{0}, h(0)=\varphi(0)=$ $0, \tilde{h}^{\prime}(0)>0$,

$$
-h\left(A_{s}^{*}(Y)\right)<-U^{\prime}\left(r_{0}-k\right)\left(\varphi\left(A_{s}^{*}(Y)\right)\right) \quad d s \otimes d \mathbb{P} \quad \text { a.e. when } \quad A_{s}>0 .
$$

For any control $\left(R, A^{*}(Y)\right) \in \mathcal{A}$ that is not identical to $\left(r_{0}, 0\right)$,

$$
\begin{aligned}
J_{0}^{C}\left(R, A^{*}(Y)\right) & =\mathbb{E}\left(\int_{0}^{\infty} e^{-\delta s}\left(U\left(R_{s}-k\right)-h\left(A_{s}^{*}(Y)\right)\right) d s\right) \\
& <\mathbb{E}\left(\int_{0}^{\infty} e^{-\delta s}\left(U\left(r_{0}-k\right)+U^{\prime}\left(r_{0}-k\right)\left(R_{s}-\varphi\left(A_{s}^{*}(Y)\right)-r_{0}\right) d s\right)\right. \\
& <\frac{U\left(r_{0}-k\right)}{\delta}-U^{\prime}\left(r_{0}-k\right)\left(\frac{r_{0}-k}{\delta}+\mathbb{E}\left(\int_{0}^{\infty} e^{-\delta s}\left(\varphi\left(A_{s}^{*}(Y)\right)-R_{s}+k\right) d s\right)\right),
\end{aligned}
$$

where the last inequality holds from (3.6) and (3.7). Since $r_{0}=U^{-1}\left(\delta V_{0}^{C}\right)+k$, for $(R, A) \neq$ $\left(r_{0}, 0\right)$,

$$
\delta J_{0}^{C}(R, A)<\delta V_{0}^{C}-U^{\prime}\left(r_{0}-k\right)\left[r_{0}-k+\delta J_{0}^{P}(R, A)\right] .
$$

By taking the supremum over all admissible strategies, we get

$$
\delta V_{0}^{P} \leq k-r_{0}<0
$$

On the other hand, by Lemma 3.1, one has $V_{0}^{P} \geq 0$. This gives a contradiction to (3.9), and $V_{0}^{C}$ is necessarily smaller than $\frac{1}{\delta}\left(U o\left(U^{\prime}\right)^{-1}\left(\frac{h^{\prime}(0)}{\varphi^{\prime}(0)}\right)\right)$.

ii) We now prove that $v(\bar{x})=0$.

The proof follows the same lines as the previous part $i)$ with this time $V_{0}^{C}=\bar{x}=\frac{1}{\delta}\left(U o\left(U^{\prime}\right)^{-1}\left(\frac{h^{\prime}(0)}{\varphi^{\prime}(0)}\right)\right)$. We notice that the constant control $\left(r_{0}, 0\right)$, which leads to $J_{0}^{C}\left(r_{0}, 0\right)=\bar{x}$, is an admissible control and so the equality $V_{0}^{C}=\bar{x}$ makes sense. Let us recall that $r_{0}=k+U^{-1}\left(\delta V_{0}^{C}\right)$, $U^{\prime}\left(r_{0}-k\right) \varphi^{\prime}(0)=h^{\prime}(0)$. For any control $\left(R, A^{*}(Y)\right) \in \mathcal{A}$, one has by concavity of the function $U$

$$
U\left(R_{s}-k\right) \leqslant U\left(r_{0}-k\right)+U^{\prime}\left(r_{0}-k\right)\left(R_{s}-r_{0}\right), \quad d s \otimes d \mathbb{P} \quad \text { a.e. }
$$

and by convexity of $\tilde{h}$

$$
-h\left(A_{s}^{*}(Y)\right) \leqslant-U^{\prime}\left(r_{0}-k\right)\left(\varphi\left(A_{s}^{*}(Y)\right)\right) \quad d s \otimes d \mathbb{P} \quad \text { a.e. }
$$


thus

$$
J_{0}^{C}\left(R, A^{*}(Y)\right) \leqslant \frac{U\left(r_{0}-k\right)}{\delta}-U^{\prime}\left(r_{0}-k\right)\left(\frac{r_{0}-k}{\delta}+\mathbb{E}\left(\int_{0}^{\infty} e^{-\delta s}\left(\varphi\left(A_{s}^{*}(Y)\right)-R_{s}+k\right) d s\right)\right)
$$

and $r_{0}=k+U^{-1}(\delta \bar{x})$ implies

$$
\delta J_{0}^{C}\left(R, A^{*}(Y)\right) \leqslant \delta \bar{x}-U^{\prime}\left(r_{0}-k\right)\left[r_{0}-k+\delta J_{0}^{P}\left(R, A^{*}(Y)\right)\right] .
$$

Taking the supremum over all incentive compatible admissible strategies, we get

$$
\delta v\left(V_{0}^{C}\right) \leq k-r_{0} \leq 0 \text {. }
$$

Besides $v \geqslant 0$ by Lemma 3.1. Therefore $v(\bar{x})=0$.

Lemma 3.5 The function $v$ satisfies

$$
v(0)=\frac{1}{\delta} \sup _{0 \leq x \leq \bar{r}-k}\left(\varphi \circ h^{-1} \circ U(x)-x\right)
$$

Proof: Since every admissible control $\left(R, A^{*}(Y)\right) \in \mathcal{A}$ must satisfy $U\left(R_{s}-k\right)-h\left(A^{*}(Y)_{s}\right) \geq$ $0, d s \otimes d \mathbb{P}$ a.e, then the equality $V_{0}^{C}=0$ holds for every control $\left(R^{*}, A^{*}(Y)\right) \in \mathcal{A}$ satisfying $U\left(R_{s}^{*}-k\right)-h\left(A^{*}(Y)_{s}\right)=0, d s \otimes d \mathbb{P}$ a.e which is equivalent to

$$
h^{-1} \circ U\left(R_{s}^{*}-k\right)=A^{*}(Y)_{s}, d s \otimes d \mathbb{P} \text { a.e. }
$$

In this class of controls, one has

$$
\varphi \circ h^{-1} \circ U\left(R_{s}^{*}-k\right)-R_{s}^{*}+k \leq \sup _{k \leq r \leq \bar{r}}\left(\varphi \circ h^{-1} \circ U(r-k)-(r-k)\right) d s \otimes d \mathbb{P} \text { a.e. }
$$

This shows that

$$
V_{0}^{P} \leq \frac{1}{\delta} \sup _{k \leq r \leq \bar{r}}\left(\varphi \circ h^{-1} \circ U(r-k)-(r-k)\right)
$$

and the equality holds when $\left(R_{s}^{*}, A^{*}(Y)_{s}\right)=(\hat{r}, \hat{a}) d s \otimes d \mathbb{P}$ a.e. where $\hat{r}$ realizes the maximum of the function $r \longrightarrow\left(\varphi \circ h^{-1} \circ U(r-k)-(r-k)\right)$ on $[k, \bar{r}]$ and $\hat{a}=h^{-1} \circ U(\hat{r}-k)$. We easily check that the control $(\hat{r}, \hat{a})$ is indeed admissible (incentive compatible). This proves (3.11).

We now proceed to the verification Theorem (cf. [16] Th. 3.5.3., infinite horizon). Thanks to Proposition 3.4 and Lemma 3.5, we study the HJB equation on a bounded domain $[0, \bar{x}]$ with the Dirichlet boundary conditions

$$
v(0)=\frac{1}{\delta} \sup _{0 \leq x \leq \bar{r}-k}\left(\varphi \circ h^{-1} \circ U(x)-x\right), \quad v(\bar{x})=0 .
$$


Theorem 3.6 Under Assumption 2.2 the HJB equation (3.1) with boundary conditions (3.12) admits a unique solution $w$ in $C^{2}((0, \bar{x})) \cap C^{0}([0, \bar{x}])$. Let $\left(a^{*}(x), r^{*}(x)\right)$ be the argmax in the HJB equation (3.1). We assume that $a^{*}$ is of bounded variation, then the associated stochastic differential equation

$$
d V_{t}^{C}=\delta V_{t}^{C} d t-\left(U\left(r^{*}\left(V_{t}^{C}\right)-k\right)-h\left(a^{*}\left(V_{t}^{C}\right)\right) d t+\sigma \frac{h^{\prime}\left(a^{*}\left(V_{t}^{C}\right)\right.}{\varphi^{\prime}\left(a^{*}\left(V_{t}^{C}\right)\right)} d W_{t}, V_{0}^{C}=x\right.
$$

admits a unique strong solution denoted as $V^{C}$. We define the following controls:

$A_{t}^{*}:=a^{*}\left(V_{t}^{C}\right), R_{t}^{*}:=r^{*}\left(V_{t}^{C}\right) d t \otimes d \mathbb{P}$ almost everywhere. Then, $\left(R^{*}, A^{*}\right)$ is the optimal control in $\mathcal{A}$. As a conclusion, the public value function satisfies $v(x)=w(x)$.

Remark that on the numerical simulations, $a^{*}$ is decreasing and thus is indeed of bounded variation.

Proof: Step 1: The equation $(3.1)$ admits an unique $C^{2}((0, \bar{x})) \cap C^{0}([0, \bar{x}]$ solution.

This follows from Theorem 1 in [19], whose assumptions are satisfied. Indeed the set of controls $\mathcal{C}=\left\{(r, a) \in[k, \bar{r}] \times \mathbb{R}^{+}, h(a) \leq U(r-k)\right\}$ is a nonempty compact set. The coefficients of the HJB equation (3.1) are affine functions of the variable $x$, and continuous functions in the control $(r, a)$, for each $x$, thus standard linear growth assumptions on the coefficients of (3.1) are satisfied. Under Assumption 2.2, the function $\frac{h^{\prime}}{\varphi^{\prime}}$ is increasing positive, and $0<\frac{h^{\prime}(0)}{\varphi^{\prime}(0)} \leq \frac{h^{\prime}(a)}{\varphi^{\prime}(a)}$. Therefore the volatitity coefficient of the HJB equation (3.1) admits a uniform lower bound. In addition, the coefficient $-r+\varphi(a)+k$ is bounded on $\mathcal{C}$. Therefore, applying Theorem 1 in [19], the HJB equation (3.1) has a twice continously differentiable solution in $(0, \bar{x})$, and continuous on $[0, \bar{x}]$.

Furthermore Proposition 3.2 proves that for all $x$ there exists an admissible pair $\left(r^{*}(x), a^{*}(x)\right)$ in $\mathcal{C}$ such that

$$
\left.-\delta w(x)+\mathcal{L}^{\left(r^{*}(x), a^{*}(x)\right)} w(x)-r^{*}(x)+\varphi\left(a^{*}(x)\right)+k=\max _{(r, a) \in \mathcal{C}}\left[-\delta w(x)+\mathcal{L}^{r, a} w(x)-r+\varphi(a)+k\right)\right] .
$$

Step 2: $w(x) \geq v(x)$

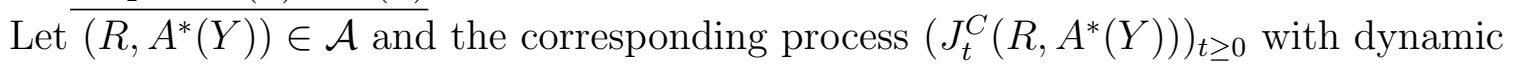
$d J_{t}^{C}\left(R, A^{*}(Y)\right)=\delta J_{t}^{C}\left(R, A^{*}(Y)\right) d t-\left(U\left(R_{t}-k\right)-h\left(A_{t}^{*}(Y)\right)\right) d t+\sigma \frac{h^{\prime}\left(A_{t}^{*}(Y)\right)}{\varphi^{\prime}\left(A_{t}^{*}(Y)\right)} d W_{t}, J_{0}^{C}\left(R, A^{*}(Y)\right)=x$

Applying Itô's formula to the process $\left(e^{-\delta t} w\left(J_{t}^{C}\left(R, A^{*}(Y)\right)\right)\right)_{t \geq 0}$

$$
\begin{aligned}
e^{-\delta T} w\left(J_{T}^{C}\left(R, A^{*}(Y)\right)\right)=w(x) & +\int_{0}^{T} e^{-\delta s}\left[-\delta w\left(J_{s}^{C}\left(R, A^{*}(Y)\right)\right)+\mathcal{L}^{R, A^{*}(Y)} w\left(J_{s}^{C}\right)\left(R, A^{*}(Y)\right)\right] d s \\
& -\int_{0}^{T} e^{-\delta s} \sigma w^{\prime}\left(J_{s}^{C}\left(R, A^{*}(Y)\right)\right) \frac{h^{\prime}\left(A_{s}^{*}(Y)\right)}{\varphi^{\prime}\left(A_{s}^{*}(Y)\right)} d W_{s}
\end{aligned}
$$

with $w^{\prime}\left(J_{t}^{C}\left(R, A^{*}(Y)\right)\right) \frac{h^{\prime}\left(A_{t}^{*}(Y)\right)}{\varphi^{\prime}\left(A_{t}^{*}(Y)\right)}$ being a bounded process. Taking the expectation, for all $T$ $E_{x}\left[e^{-\delta T} w\left(J_{T}^{C}\left(R, A^{*}(Y)\right)\right)\right]=w(x)+E_{x}\left[\int_{0}^{T} e^{-\delta s}\left[\mathcal{L}^{R_{s}, A_{s}^{*}(Y)} w\left(J_{s}^{C}\left(R, A^{*}(Y)\right)\right)-\delta w\left(J_{s}^{C}\left(R, A^{*}(Y)\right)\right)\right] d s\right]$ 
From the HJB equation 3.1$), \mathcal{L}^{R_{s}, A_{s}^{*}(Y)} w\left(J_{s}^{C}\left(R, A^{*}(Y)\right)\right)-\delta w\left(J_{s}^{C}\left(R, A^{*}(Y)\right)\right) \leq R_{s}-\varphi\left(A_{s}^{*}(Y)\right)-$ $k$ thus

$$
E_{x}\left[e^{-\delta T} w\left(J_{T}^{C}\left(R, A^{*}(Y)\right)\right)\right] \leq w(x)+E_{x}\left[\int_{0}^{T} e^{-\delta s}\left(-\varphi\left(A_{s}^{*}(Y)\right)-k+R_{s}\right) d s .\right.
$$

Using boundedness from above of $-\varphi(a)-k+r$ when $(r, a) \in \mathcal{C}$ (cf. the proof of Lemma 3.1) and admissibility conditions on $\left(R, A^{*}(Y)\right) \in \mathcal{A}$, one has $e^{-\delta s}\left|-\varphi\left(A_{s}\right)-k+R_{s}\right| \leq$ $e^{-\delta s}\left(\varphi\left(A_{s}\right)+k-R_{s}\right)^{-}+e^{-\delta s} M \in L^{1}\left(\mathbb{R}^{+} \times \Omega\right)$. By the dominated convergence theorem, we obtain

$$
\lim _{T \longrightarrow \infty} E_{x}\left[\int_{0}^{T} e^{-\delta s}\left(-\varphi\left(A_{s}^{*}(Y)\right)-k+R_{s}\right) d s\right]=E_{x}\left[\int_{0}^{\infty} e^{-\delta s}\left(-\varphi\left(A_{s}^{*}(Y)\right)-k+R_{s}\right) d s\right] .
$$

Besides, as $w$ is a continuous function and $J_{T}^{C}$ is bounded, one has

$$
\lim _{T \longrightarrow \infty} E_{x}\left[e^{-\delta T} w\left(J_{T}^{C}\left(R, A^{*}(Y)\right)\right)\right]=0 \text { for all }\left(R, A^{*}(Y)\right) \in \mathcal{A}
$$

Therefore

$$
0 \leq w(x)+E_{x}\left[\int_{0}^{\infty} e^{-\delta s}\left(-\varphi\left(A_{s}^{*}(Y)\right)-k+R_{s}\right) d s\right],
$$

so for any $\left(R, A^{*}(Y)\right) \in \mathcal{A}$ we get

$$
w(x) \geq E_{x}\left[\int_{0}^{\infty} e^{-\delta s}\left(\varphi\left(A_{s}^{*}(Y)\right)+k-R_{s}\right) d s\right]
$$

and

$$
w(x) \geq \sup _{\left(R, A^{*}(Y)\right) \in \mathcal{A}} V_{0}^{P}\left(R, A^{*}(Y)\right)=v(x) .
$$

Step 3: the SDE (3.13) admits a unique strong solution

Let us consider the SDE (3.13) associated to the optimal pair $\left(r^{*}\left(V_{t}^{C}\right), a^{*}\left(V_{t}^{C}\right)\right)$, then $\tilde{V}_{t}^{C}:=$ $e^{-\delta t} V_{t}^{C}, d t \otimes d \mathbb{P}$ a.e. satisfies the $\mathrm{SDE}$

$$
d \tilde{V}_{t}^{C}=-e^{-\delta t}\left(U\left(r^{*}\left(V_{t}^{C}\right)-k\right)-h\left(a^{*}\left(V_{t}^{C}\right)\right) d t+\sigma e^{-\delta t} \frac{h^{\prime}\left(a^{*}\left(V_{t}^{C}\right)\right.}{\varphi^{\prime}\left(a^{*}\left(V_{t}^{C}\right)\right)} d W_{t}, V_{0}^{C}=x .\right.
$$

The existence of a strong solution of this SDE is given by Nakao [14] (Theorem p. 516), as the drift is bounded measurable, the volatility $\sigma \frac{h^{\prime}}{\varphi^{\prime}}\left(a^{*}\right)$ is strictly bounded from below and of bounded variation (since it is the case for $a^{*}$ ). The existence of a strong solution of the SDE (3.13) follows.

Step 4: $w(x) \leq v(x)$

This solution actually is the process denoted as $\left(V_{t}^{C}\right)_{t \geq 0}$ meaning $V^{C}=J^{C}\left(R^{*}, A^{*}\right)$ where $R_{s}^{*}:=r^{*}\left(V_{s}^{C}\right), A^{*}:=a^{*}\left(V_{s}^{C}\right), d s \otimes d \mathbb{P}$ a.e.. We now repeat the above arguments of Step 2:

$$
e^{-\delta T \wedge \tau_{n}} w\left(V_{T \wedge \tau_{n}}^{C}\right)=w(x)+\int_{0}^{T \wedge \tau_{n}} e^{-\delta s}\left[-\delta w\left(V_{s}^{C}\right)+\mathcal{L}^{R_{s}^{*}, A_{s}^{*}} w\left(V_{s}^{C}\right)\right] d s-\int_{0}^{T \wedge \tau_{n}} e^{-\delta s} \sigma w^{\prime}\left(V_{s}^{C}\right) \frac{h^{\prime}\left(A_{s}^{*}\right)}{\varphi^{\prime}\left(A_{s}^{*}\right)} d W_{s} .
$$


Thus for $n$ and all $T$

$$
E_{x}\left[e^{-\delta T \wedge \tau_{n}} w\left(V_{T \wedge \tau_{n}}^{C}\right)\right]=w(x)+E_{x}\left[\int_{0}^{T \wedge \tau_{n}} e^{-\delta s}\left[-\delta w\left(V_{s}^{C}\right)+\mathcal{L}^{R_{s}^{*}, A_{s}^{*}} w\left(V_{s}^{C}\right)\right] d s\right] .
$$

But since $w$ satisfies the HJB equation (3.1) with such optimal controls we get

$$
\begin{gathered}
E_{x}\left[e^{-\delta T \wedge \tau_{n}} w\left(V_{T \wedge \tau_{n}}^{C}\right)\right]=w(x)+E_{x}\left[\int_{0}^{T \wedge \tau_{n}} e^{-\delta s}\left(R_{s}^{*}-k-\varphi\left(A_{s}^{*}\right)\right) d s\right] \\
=w(x)-E_{x}\left[\int_{0}^{T \wedge \tau_{n}} e^{-\delta s}\left(R_{s}^{*}-k-\varphi\left(A_{s}^{*}\right)\right)^{-} d s\right]+E_{x}\left[\int_{0}^{T \wedge \tau_{n}} e^{-\delta s}\left(R_{s}^{*}-k-\varphi\left(A_{s}^{*}\right)\right)^{+} d s\right] .
\end{gathered}
$$

Taking into account the boundedness of the controls $\left(r^{*}\left(V_{t}^{C}\right), a^{*}\left(V_{t}^{C}\right)\right)_{t \geq 0}$ (cf. Proposition 3.2), the dominated convergence theorem allows us to get $n$ and $T$ going to infinity in $E_{x}\left[\int_{0}^{T \wedge \tau_{n}} e^{-\delta s}\left(R_{s}^{*}-\right.\right.$ $\left.k-\varphi\left(A_{s}^{*}\right)\right) d s$. Besides, Fatou's lemma and boundedness of $w$ allow us to get $n$ and $T$ going to infinity in $E_{x}\left[e^{-\delta T \wedge \tau_{n}} w\left(V_{T \wedge \tau_{n}}^{C}\right)\right]$. Therefore

$$
w(x) \leq J_{0}^{P}\left(R^{*}, A^{*}\right) \leq v(x) .
$$

Conclusion: $w$ is the public value function $v$ defined in equation 2.12$)$ and $\left(a^{*}\left(V_{t}^{C}\right), r^{*}\left(V_{t}^{C}\right)\right)$ is a Markovian optimal control.

\subsection{Going back to the original set $\mathcal{A}^{X}$ of control processes}

In this subsection, we will prove that the process $V^{C}$ is $\mathbb{F}^{X}$-adapted.

Proposition 3.7 Under the assumptions of Theorem 3.6, the unique strong solution to the stochastic differential equation (3.13) admits an infinite explosion time, and the filtrations $\mathbb{F}^{X}$, $\mathbb{F}^{V^{C}}$ and $\mathbb{F}$ coincide at the optimum.

Proof: First, as $R$ and $A$ are bounded, the strong solution of the SDE (3.13) does not explode. Besides, $\mathbb{F}^{V^{C}}$ and $\mathbb{F}^{X}$ are obviously included in $\mathbb{F}$.

(i) We first express $V^{C}$ as the solution of the SDE (3.13)

$$
d V_{t}^{C}=\delta V_{t}^{C} d t-\left(U\left(r^{*}\left(V_{t}^{C}\right)-k\right)-h\left(a^{*}\left(V_{t}^{C}\right)\right)\right) d t+\sigma \frac{h^{\prime}}{\varphi^{\prime}}\left(a^{*}\left(V_{t}^{C}\right)\right) d W_{t} .
$$

Under the assumptions of Theorem 3.6, this SDE admits an unique strong solution, thus the filtrations generated by $W$ and $V^{C}$ coincide since $\sigma \frac{h^{\prime}}{\varphi^{\prime}}\left(a^{*}\left(V_{t}^{C}\right)\right)$ the coefficient of $d W_{t}$ is positive (cf. Corollary 1.12 of Revuz-Yor [17]).

(ii) On the other hand, by definition:

$$
d X_{t}=\left(\varphi\left(a^{*}\left(V_{t}^{C}\right)\right)+k\right) d t+\sigma d W_{t}
$$


thus, since $\sigma>0$,

$$
d W_{t}=\frac{1}{\sigma}\left[d X_{t}-\left(\varphi\left(a^{*}\left(V_{t}^{C}\right)\right)+k\right) d t\right]
$$

and

$$
d V_{t}^{C}=\delta V_{t}^{C} d t-\left(U\left(r^{*}\left(V_{t}^{C}\right)-k\right)-h\left(a^{*}\left(V_{t}^{C}\right)\right) d t+\frac{h^{\prime}}{\varphi^{\prime}}\left(a^{*}\left(V_{t}^{C}\right)\right)\left(-\left(\varphi\left(a^{*}\left(V_{t}^{C}\right)\right)+k\right) d t+d X_{t}\right) .\right.
$$

Once again, under the assumptions of Theorem 3.6, $V^{C}$ is a strong solution of this stochastic differential equation driven by $X$ so this process $V^{C}$ is $\mathbb{F}^{X}$-adapted. Therefore the three filtrations $\mathbb{F}^{V^{C}}, \mathbb{F}^{X}$ and $\mathbb{F}$ coincide at the optimum.

\section{Numerical implementation}

The consortium continuation value is the state parameter used in the resolution of the stochastic control problem of Section 3. Proposition 3.4 gives an upper bound for the consortium initial value $V_{0}^{C} \in[0, \bar{x}]$.

\subsection{Howard's Algorithm}

The HJB-equation (3.1) is written as follows

$$
\sup _{(r, a) \in \mathcal{C}}\left[-\delta v(x)+v^{\prime}(x)(\delta x-U(r-k)+h(a))+\frac{1}{2} v^{\prime \prime}(x)\left(\sigma \frac{h^{\prime}(a)}{\varphi^{\prime}(a)}\right)^{2}-r+\varphi(a)+k\right]=0 .
$$

Let $\Delta$ be the finite difference step on the state coordinate and $\left(x_{i}\right)_{i=1, N}, x_{i}=i \Delta$, be the points of the grid $\Omega_{\Delta}$. The equation (4.1) is discretized by replacing the first and second derivatives of $v$ with the following approximations

$$
\begin{gathered}
v^{\prime}(x) \simeq \begin{cases}\frac{v(x+\Delta)-v(x)}{\frac{v(x)-\hat{v}(x-\Delta)}{\Delta}} & \text { if } \delta x+h(a)-U(r-k) \geq 0\end{cases} \\
v^{\prime \prime}(x) \simeq \frac{v(x+\Delta)-2 v(x)+v(x-\Delta)}{\Delta^{2}} . \\
v(0)=\frac{1}{\delta} \sup _{0 \leq x \leq \bar{r}-k} \varphi \circ h^{-1} \circ U(x)-x, v(\bar{x})=0 .
\end{gathered}
$$

where $\bar{x}=\frac{1}{\delta}\left(U o\left(U^{\prime}\right)^{-1}\left(\frac{h^{\prime}(0)}{\varphi^{\prime}(0)}\right)\right)=N \Delta$.

This leads to the system of $(N-1)$ equations with $(N-1)$ unknowns $\left(v^{\Delta}\left(y_{i}\right)\right)_{i=1, \ldots N-1}$ :

$$
\max _{(r, a) \in \mathcal{C}}\left[A^{\Delta,(r, a)} v^{\Delta}\left(x_{i}\right)+B^{\Delta,(r, a)}\right]=0
$$


where $B^{\triangle,(r, a)}$ is given by

$$
B^{\triangle,(r, a)}=\left(\begin{array}{c}
-r+\varphi(a)+k+\left(\frac{b^{-}(\Delta)}{\Delta}+\frac{a(\Delta}{\Delta^{2}}\right) v(0) \\
-r+\varphi(a)+k \\
\vdots \\
\vdots \\
-r+\varphi(a)+k
\end{array}\right)
$$

the matrix $A^{\Delta,(r, a)}$ is defined as follows:

$\left[A^{\Delta,(r, a)}\right]_{i, i-1}=\frac{b^{-}\left(y_{i}\right)}{\Delta}+\frac{a\left(x_{i}\right)}{\Delta^{2}} ;\left[A^{\Delta,(r, a)}\right]_{i, i}=c\left(y_{i}\right)-\frac{\left|b\left(x_{i}\right)\right|}{\Delta}-2 \frac{a\left(x_{i}\right)}{\Delta^{2}} ;\left[A^{\Delta,(r, a)}\right]_{i, i+1}=\frac{b^{+}\left(x_{i}\right)}{\Delta}+\frac{a\left(x_{i}\right)}{\Delta^{2}} ;$

with $b^{+}(x)=\max (b(x), 0), b^{-}(x)=\max (-b(x), 0)$ and

$$
\begin{aligned}
c(x) & =-\delta \\
b(x) & =(h(a)-U(r-k))+\delta x, \\
a(x) & =\frac{1}{2}\left(\sigma \frac{h^{\prime}(a)}{\varphi^{\prime}(a)}\right)^{2} .
\end{aligned}
$$

To solve the latter equation we use an iterative Howard algorithm (cf. Howard [13] chapter 8). It consists in computing two sequences $\left(r^{n}\left(x_{i}\right), a^{n}\left(x_{i}\right)\right)_{i=1, \ldots N-1}$ and $\left(v^{\Delta, n}\left(y_{i}\right)\right)_{i=1, \ldots N-1}$ (starting from $\left(r^{1}, a^{1}\right)$ chosen arbitrary):

- step $2 n-1$ : to the strategy $\left(r^{n}, a^{n}\right)$ we compute $v^{\Delta, n}$ solution of the linear system

$$
A^{\Delta,\left(r^{n}, a^{n}\right)} v^{\Delta}+B^{\Delta,\left(r^{n}, a^{n}\right)}=0
$$

on the grid $\Omega^{\Delta}$.

- step $2 n: v^{\Delta, n}$ is associated with a strategy

$$
\left(r^{n+1}, a^{n+1}\right) \in \arg \max _{(r, a) \in \mathcal{C}}\left(A^{\Delta,(r, a)} v^{\Delta, n}+B^{\Delta,(r, a)}\right) .
$$

The convergence of the Howard algorithm holds when the matrix $A^{\Delta,(r, a)}$ satisfies the discrete maximum principle: a sufficient condition is that $A^{\Delta,(r, a)}$ is diagonally dominant. This is the case since $c(x)<0$.

\subsection{Effort and rent}

We recall $r^{*}(x)=\left(k+\left(U^{\prime}\right)^{-1}\left(\frac{-1}{v^{\prime}(x)}\right) \mathbf{1}_{v^{\prime}(x)<0}\right) \wedge \bar{r}$

and $a^{*}(x)=\arg \max \left(a \rightarrow v^{\prime}(x) h(a)+v^{\prime \prime}(x) \sigma^{2} \psi(a)+\varphi(a)\right)$

in the compact interval $\left.\left[0, h^{-1} \circ U\left(\left(U^{\prime}\right)^{-1}\left(\frac{-1}{v^{\prime}(x)}\right) \mathbf{1}_{v^{\prime}(x)<0}\right) \wedge(\bar{r}-k)\right)\right]$.

If $v^{\prime}(x) \geq 0 r^{*}(x)=k$, and $a^{*}(x)=\arg \max \left(a \rightarrow v^{\prime}(x) h(a)+v^{\prime \prime}(x) \sigma^{2} \psi(a)+\varphi(a)\right)$ in the compact interval $\left[0, h^{-1}(0)\right]=\{0\}$.

If $v^{\prime}(x)<0 r^{*}(x)=\left(k+\left(U^{\prime}\right)^{-1}\left(\frac{-1}{v^{\prime}(x)}\right)\right) \wedge \bar{r}$, and $a^{*}(x)=\arg \max \left(a \rightarrow v^{\prime}(x) h(a)+\right.$ $\left.v^{\prime \prime}(x) \sigma^{2} \psi(a)+\varphi(a)\right)$ in the compact interval $\left[0, h^{-1} \circ U\left(\left(\left(U^{\prime}\right)^{-1}\left(\frac{-1}{v^{\prime}(x)}\right) \mathbf{1}_{v^{\prime}(x)<0}\right) \wedge(\bar{r}-k)\right)\right]$. 


\subsection{Numerical results}

In this section we choose $U(x)=\sqrt{x}$ and the functions of the example $3.3: \varphi(x)=x+\ln (1+x)$, $h(x)=\frac{2}{3} \sqrt{1+x}(x+4)-\frac{8}{3}$, so $2 \psi(x)=1+x$.

We choose the parameter $\delta=0.1$. We restrict our figures to $x \in\left[0, \frac{1}{\delta}\left(U o\left(U^{\prime}\right)^{-1}\left(\frac{h^{\prime}(0)}{\varphi^{\prime}(0)}\right)\right)\right](\mathrm{cf}$. Proposition 3.4 , that is $[0,5]$ in our numerical example.

In Figure 2, Figure 3 and Figure $4, k=2$ and $\sigma$ varies : $\sigma=0.5,0.8$ or 1 . Figure 1 gives the optimal rent function of the optimal effort, for $k=2$ and $\sigma=0.8$.

We observe that $v$ and $a^{*}$ are non-increasing functions of the consortium value.

\subsubsection{Graph of the optimal rent $r$ as a function of $a$}

The more interesting observation is that the optimal rent is an increasing convex function of the optimal effort. This contradicts the usual assumption of linear dependence between rent and effort. Besides, the qualitative behaviour of these optimal parameters is the same with respect to both $\sigma$ and $k$.

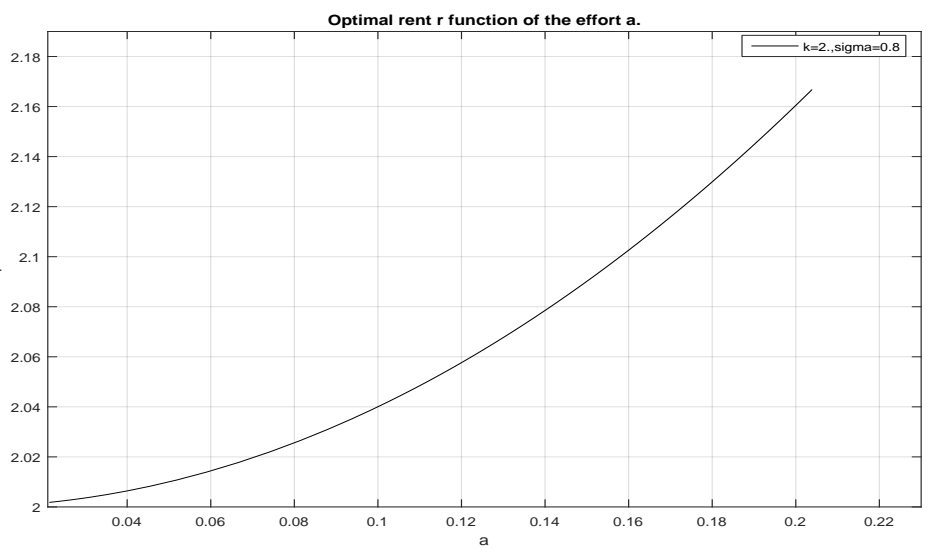

Figure 1: Optimal rent $r^{*}$ function of the effort $a^{*}$. 


\subsubsection{Sensibility of the results to parameter $\sigma$}

According to Figure 2, it seems that the optimal public value function is increasing with respect to $\sigma$ : the risk is supported by the consortium. The same behavior is observed for the optimal effort (Figure 3) and for the optimal rent (Figure 4) in case of $x$ large enough. But, when $x$ is lower the optimal effort is decreasing. In case of a low level of the private continuation value $x$, the consortium is not ready to provide more efforts. This behavior is observed for any parameter $k$.

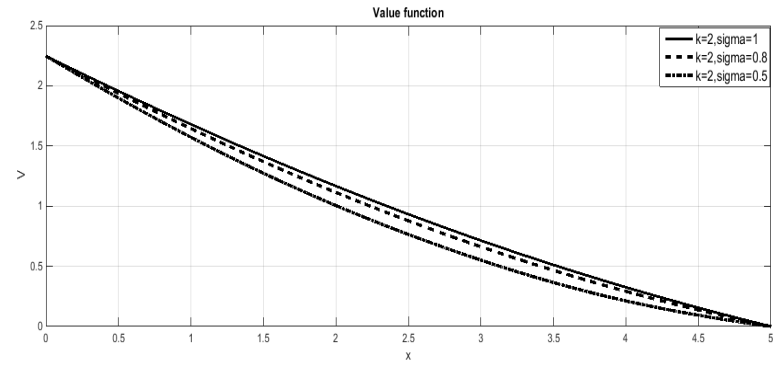

Figure 2: Value function $v$ for different $\sigma$.

\subsubsection{Sensibility of the results to parameter $k$}

Actually, the true parameter control is $r-k$, so, as it could be expected, the parameter $k$ has no impact on the behaviors of $v, r$, and $a$. 


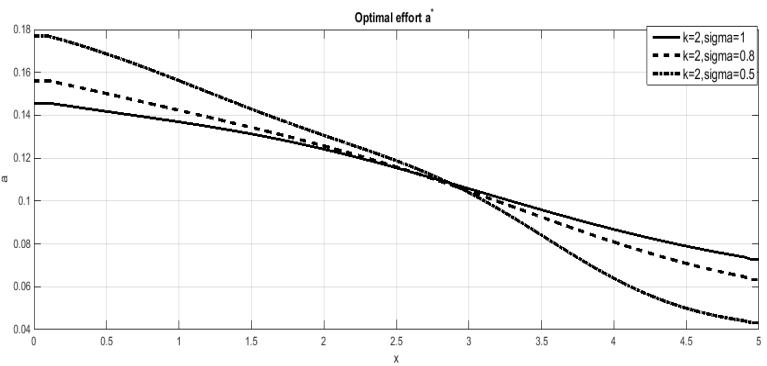

Figure 3: optimal effort $a^{*}$ for different $\sigma$.

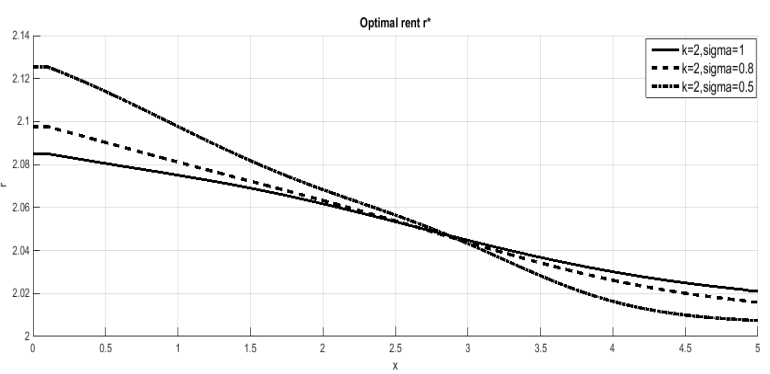

Figure 4: Optimal rent $r^{*}$, for different $\sigma$. 


\section{Conclusion}

This paper provides a characterisation of optimal public private partnership contracts in a moral hazard framework, using martingale methods and stochastic control. A numerical example shows that, in particular, the optimal rent is a convex (and not linear) function of the effort. This convexity, due to the information asymmetry between the consortium and the public entity, implies for the public entity a more and more costly contract to encourage the consortium to do more efforts. This feature should be taken into account in the models concerning PPP contracts.

\section{Appendix A}

We need to know some sufficient conditions to get the cost process $\left(C_{s}\right)$ non-negative on a time interval $[0, T]$. Recall the Inverse Gaussian law $(I G(\lambda, \mu))$ with density on $\mathbb{R}^{+}$

$$
f(t, \mu, \lambda)=\left(\frac{\lambda}{2 \pi t^{3}}\right)^{\frac{1}{2}} \exp \left(-\frac{\lambda}{2 \mu^{2} t}(t-\mu)^{2}\right) .
$$

The cost process $C$ is a drifted Brownian motion and the event

$$
\left\{\inf _{0 \leq s \leq T} C_{s} \geq 0\right\}=\left\{\inf _{0 \leq s \leq T} k s+\sigma W_{s} \geq-C_{0}\right\}=\left\{\sup _{0 \leq s \leq T}\left(-k s-\sigma W_{s}\right) \leq C_{0}\right\}=\left\{T_{C_{0}}>T\right\}
$$

where $T_{C_{0}}$ is an hitting time. It is well known (cf. [6] for instance) that the law of $T_{C_{0}}$ is $I G\left(-\frac{C_{0}}{k}, \frac{C_{0}^{2}}{\sigma^{2}}\right)$ meaning that we would like to bound with 0.05 (for instance)

$$
\mathbb{P}\left\{\inf _{0 \leq s \leq T} C_{s} \leq 0\right\}=\int_{0}^{T} \frac{C_{0}}{\sigma \sqrt{2 \pi t^{3}}} \exp \left(-\frac{k^{2}}{2 \sigma^{2} t}\left(t+\frac{C_{0}}{k}\right)^{2}\right) d t=\int_{0}^{T} \frac{C_{0}}{\sigma \sqrt{2 \pi t^{3}}} \exp \left(-\frac{\left(k t+C_{0}\right)^{2}}{2 \sigma^{2} t}\right) d t .
$$

After the change of variable $x^{2}=\frac{C_{0}^{2}}{t \sigma^{2}}$, since $k>0$ this probability is bounded by

$$
\left.\int_{0}^{T} \frac{C_{0}}{\sigma \sqrt{2 \pi t^{3}}} \exp \left(-\frac{C_{0}^{2}}{2 \sigma^{2} t}\right)\right) d t=2 \int_{C_{0} /(\sigma \sqrt{T})}^{\infty} \frac{1}{\sqrt{2 \pi}} \exp \left(-\frac{x^{2}}{2}\right) d x=\Phi\left(C_{0} /(\sigma \sqrt{T})\right)
$$

where $\Phi$ is the distribution function of the standard Gaussian law. A sufficient condition to get the cost non-negative on time interval $[0, T]$ with at least probability 0.95 is

$$
C_{0} /(\sigma \sqrt{T}) \geq 1.96
$$




\section{References}

[1] E. Auriol, P.M. Picard. A theory of BOT concession contracts. Journal of of Economic Behaviour and Organization, 2834, (2011).

[2] A. Bensoussan. Stochastic control of partially observable systems, Cambridge University Press, (1992).

[3] B. Biais, T. Mariotti, J.C. Rochet, S. Villeneuve. Large risks, limited liability and dynamic moral hazard. Econometrica, Vol. 78, No. 1 (January, 2010), 73-118.

[4] J. Cvitanic, J. Zhang. Contract Theory in Continuous-Time Models, Springer (2013).

[5] J. Cvitanic, D. Possamaï, N. Touzi. Moral hazard in dynamic risk management, arXiv:1510.07111 (2015).

[6] M. Chesney, M. Jeanblanc, M. Yor. Mathematical Methods for Financial Markets, Springer (2009).

[7] N. El Karoui. Les aspects probabilistes du contrôle stochastique, Saint Flour 1979, Volume 876 of Lectures Notes In Math. (1981).

[8] G.E. Espinosa, C. Hillairet, B. Jourdain, M. Pontier. Reducing the debt: Is it optimal to outsource an investment? (2016). a voir To appear in Mathematics and Financial Economics.

[9] W. Fleming and R. Rishel. Deterministic and Stochastic Optimal Control, SpringerVerlag, (1975).

[10] C. Hillairet, M. Pontier. A Modelisation of Public Private Parternships with failure time, Laurent Decreusefond and Jamal Najim ed. Springer Proceedings in Mathematics and Statistics Vol 22, 91-117, (2012).

[11] E. Iossa, D. Martimort, J. Pouyet. Partenariats Public-Privé, quelques réflexions. Revue économique, 59 (3) (2008).

[12] N. Krylov. Nonlinear Elliptic and Parabolic Equation of Second Order, D.Reidel, Boston, (1987).

[13] R. Howard. Dynamic Programming and Markov Processes. MIT Press, Cambridge, (1960).

[14] S. Nakao. On the pathwise uniqueness of solutions of one-dimensional stochastic differential equations. Osaka J. Math., 9, 513-518, (1972).

[15] H. Pagès, D. Possamaï. A mathematical treatment of bank monitoring, Finance and Stochastics, 18(1), 39-73, (2014). 
[16] H. Pham. Continuous-time stochastic control and optimization with financial applications, Series Stochastic Modeling and Applied Probability, vol 61, Springer, (2009).

[17] D. Revuz and M. Yor. Continuous Martingales and Brownian Motion, Springer, 2004.

[18] Y. Sannikov. A continuous-time version of the principal-agent problem, Rev. Econ. Studies 75 957-984, (2008).

[19] B. Strulovici, M. Szydlowski. On the smoothness of value functions and the existence of optimal strategies in diffusion models, Journal of Economic Theory 1016-1055, (2015). 